ISSN: 0213-3563

https://doi.org/10.14201/azafea202123307330

\title{
ÉTICA Y TURISMO EN EL MARCO DE LAS 'TRIBUS \\ MORALES’: EL POTENCIAL MORAL INDIRECTO \\ DE LA ACTIVIDAD TURÍSTICA DESDE \\ UNA PERSPECTIVA EVOLUTIVA
}

Ethics and Tourism in the Framework of 'Moral Tribes':

The Indirect Moral Potential of Tourism Activity

from an Evolutionary Perspective

E. Joaquín SuÁREZ-Ruíz

Universidad Nacional de La Plata

CONICET (FaHCE-UNLP)

Enviado: 30 de mayo de 2021

Aceptado: 7 de julio de 2021

\section{RESUMEN}

El objetivo de este trabajo es analizar una concepción del vínculo entre ética y turismo que puede ser pensada como alternativa a la predominante en la teoría del turismo actual. Para ello, se partirá de la perspectiva evolutiva de la moral de dos filósofos contemporáneos: Peter Singer y Joshua Greene. Según se argumentará, paralelamente a la ética del turismo y al turismo ético, también puede hablarse de una potencialidad moral del turismo cultural de masas, la cual no surge de una aplicación de preceptos éticos al turismo, sino que es inherente a este último.

Palabras clave: turismo ético; ética del turismo; turismo cultural; turismo de masas; ética evolutiva.

\section{ABSTRACT}

The objective of this article is to analyse a conception of the link between ethics and tourism that can be thought of as an alternative to the predomi- 
nant one in current tourism theory. To do this, we will start from the evolutionary perspective of morality of two contemporary philosophers: Peter Singer and Joshua Greene. As it will be argued, parallel to the ethics of tourism and ethical tourism, one can also speak of a moral potentiality of mass cultural tourism, which does not arise from an application of ethical precepts to tourism, but is inherent to the latter.

Keywords: ethical tourism; tourism ethics; cultural tourism; mass tourism; evolutionary ethics.

\section{INTRODUCCIÓN}

Según afirman especialistas (p. ej. Gössling et al., 2020; Higgins-Desbiolles, 2020a 2020b; Brouder, 2020; Niewiadomski, 2020), la pandemia de COVID-19 generará múltiples cambios en las características de la actividad turística. De allí que, en la actualidad, una de las líneas de investigación fundamentales vinculada con dicha actividad sea la articulación entre la ética y el turismo.

Aunque actualmente esta distinción se encuentra ya muy matizada, como punto de partida analítico aún es posible sostener que el turismo posee dos dimensiones generales en su constitución, una de tipo industrial y otra de tipo ético-social. La primera se relaciona con el hecho de que el turismo se ha transformado en un importante motor económico para los Estados, lo cual incentiva la búsqueda de estrategias que favorezcan su rentabilidad económica. La segunda se relaciona con otro componente inherente a esta actividad, a saber, el vínculo social que se genera entre forasteros y comunidades de diversas culturas a través de la actividad turística. A su vez, al interior del aspecto social se encuentra la faceta ética del turismo, esto es, las diferentes implicaciones morales que acarrea dicha actividad.

Vale resaltar que ni la dimensión social ni la existencia de problemas de tipo ético en el turismo son fenómenos nuevos. Como bien se resume en Tearfund:

El turismo no es sólo una transacción económica o una serie de actividades que pueden aislarse de la vida cotidiana o de su impacto en las personas. El hecho de que viajemos a otra cultura y tengamos contacto directo con las personas de allí, plantea una serie de problemas éticos: ‘acaso los locales quieren que los turistas los visiten?, ¿cuáles son las condiciones de trabajo en la industria del turismo?, ¿qué cambio genera el turismo en las relaciones y los 
valores familiares?, ¿a dónde va el dinero?, ¿quién se beneficia?, ¿cuáles son las consecuencias ambientales del turismo?, ¿ viajar a un lugar en particular apoya la democracia y los derechos humanos, o los socava?' (Tearfund, 2000, 5).

No obstante, lo que sí es relativamente reciente es su consideración como tópico de investigación académica (Lovelock y Lovelock, 2013). De hecho, si bien hasta entrado el siglo XX la faceta predominante fue la industrial, actualmente existe un gran énfasis en el aspecto social y ético del turismo. Un componente importante para el surgimiento de este énfasis fue el denominado 'giro crítico del turismo' (Ateljevic et al., 2011). Evidencia de ello son la emergencia de perspectivas como el pro-poor tourism (PPT), el turismo sustentable y el turismo voluntario, las cuales serán definidas más adelante.

En general, estas perspectivas buscan un punto de vista holístico de la actividad turística que permita escapar a la opción dicotómica, es decir, un enfoque que contemple la faceta industrial inherente a ella y, a su vez, que sea crítica con la reducción del turismo a una actividad lucrativa, para así incluir en sus análisis cuestiones sobre cómo la cultura y el poder poseen influencia en esta práctica (Cheong y Miller, 2000; Davis, 2001). Una definición del turismo desde este punto de vista amplio sería, por ejemplo, el de Teo, quien lo define como "una red compleja e intrincada de estructuras, agentes e interacciones que entrelaza las condiciones externas con las locales de manera cooperativa y competitiva" $(2003,460)$.

En relación con la dimensión ética en particular, actualmente existe la disciplina denominada 'ética del turismo', definida por Fennell como un análisis de qué está bien o mal, qué es lo correcto y lo incorrecto en la actividad turística (Fennell, 2006). En gran parte de la literatura, la ética del turismo se manifiesta como una ética aplicada, es decir, como un análisis de cómo los criterios normativos de las éticas tradicionales se aplican al turismo. Por ejemplo, a través del análisis de normas de conducta del turista a la luz de una perspectiva deontológica (Duffy, 2004, 85), de la aplicación del criterio utilitarista de la maximización imparcial de la felicidad a determinada práctica turística (Lovelock y Lovelock, 2013, 24) o de la búsqueda del término medio entre dos extremos para así dirigir el comportamiento del turista según la ética de la virtud aristotélica (Fennell, 2007, 100). La forma más común en que se manifiesta esta visión tradicional del vínculo entre ética y turismo es a través de, a nivel general, los códigos de ética (el UNWTO Global Code of

1. Las citas de habla inglesa son traducciones propias. 
Ethics es, quizás, el más importante) y de, a nivel particular, los códigos de conducta.

Otra disciplina relacionada es el 'turismo ético', la cual incluye una variedad de perspectivas como las tres mencionadas más arriba, cuyo propósito general es "crear beneficios sociales, culturales y económicos para la población local de destino y minimizar el desvío de fondos (...). Su fin último es cambiar los patrones de consumo en el primer mundo" (Mowforth y Munt, 2016, 103). De modo que, mientras la ética del turismo puede comprenderse como una ética aplicada al turismo, el turismo ético representa un paradigma emergente del énfasis contemporáneo en la dimensión ético-social de la actividad turística, en el cual los objetivos no son sólo el rédito económico sino también cuestiones como el cuidado del medio ambiente y las acciones solidarias para con los menos favorecidos.

Más allá de la relevancia del nuevo énfasis en la dimensión ético-social del turismo, numerosos autores afirman que existen varios problemas relacionados con la idea de normatividad moral, es decir, de aquello que permite determinar la corrección o incorrección a nivel moral, que predomina en esta corriente actual. La mayoría de sus problemas surgirían, justamente, de la constitución dual del turismo: siendo que, en la práctica, el turismo continúa siendo una actividad preeminentemente industrial, es decir, una cuyo fin principal es el rédito económico, las pretensiones normativas suelen quedar relegadas al ámbito de las obligaciones formales, sin un impacto importante en lo concreto. Por ejemplo, Moufakkir, respecto de esta 'actitud moralizante' en la teoría del turismo, afirma que "la ironía de las ironías es que esta moralización habita en libros y en revistas, pero rara vez alcanza su objetivo. (...) La mayoría de los consumidores no tienen absolutamente ningún conocimiento sobre esta moralización del turismo ético o de la ética en el turismo" $(2012,12)$.

Es por ello que, paradójicamente, en la actualidad parece haber surgido una suerte de crítica de la crítica, según la cual en las interpretaciones más extendidas del giro crítico predomina una visión que, procurando ser novedosa, termina reproduciendo vicios antiguos. Otro teórico, Jim Butcher, caracteriza este pretendido 'turismo moral nuevo' como un "exceso de equipaje moral” que "formaliza el sentido común” $(2003,71)$, más que como un dispositivo regulador efectivo. También es pertinente tener en cuenta estudios como los de la investigadora Stroma Cole, quien afirma que herramientas como los códigos de conducta perjudican la experiencia del turista, por el hecho de que en lugar de estimular el aprendizaje de cómo relacionarse y 
respetar las costumbres de la cultura local desconocida, terminan caracterizando a los individuos como principiantes que no pueden reconocerlas por sí mismos (2007, 444). A su vez, también según Butcher (2014), esta concepción tradicional de la ética en el turismo corre el riesgo de poner trabas en el cambio a nivel socio-político.

Entonces parece existir en el giro crítico del turismo una dificultad que persiste más allá de la búsqueda de renovación teórica. La hipótesis que se sostendrá en este trabajo es que el núcleo problemático en cuestión es la visión tradicional del vínculo entre la ética y el turismo, basado generalmente en la aplicación de los criterios abstractos de las éticas normativas tradicionales a la teoría del turismo. Dicho problema no sólo abarca a la ética del turismo, sino también, tal como se argumentará en la sección siguiente, al turismo ético.

Habiendo evidenciado los problemas ligados a la concepción tradicional del vínculo entre ética y turismo, en este artículo se realizará una exploración de dicho vínculo desde un punto de vista filosófico alternativo. El argumento central reside en que la actividad turística, sobre todo en lo que respecta al turismo cultural de masas (término que será definido más adelante), siendo que se caracteriza, entre otras cosas, por una interacción entre comunidades culturalmente diversas (por sus valores morales, religiosos, estéticos, etc.), favorece condiciones propicias para el surgimiento de vínculos empáticos potencialmente relevantes a nivel moral.

En comparación con los abordajes tradicionales mencionados más arriba, la diferencia principal de esta investigación es el hecho de que se priorizará un enfoque descriptivo. Es decir, el objetivo no será realizar una aplicación de criterios normativos predeterminados a la actividad turística, sino, más bien, sacar a la luz el potencial moral indirecto latente en el turismo cultural de masas, el cual no necesariamente entra en contradicción con la dimensión industrial de esta actividad. Ahora bien, más allá de que el abordaje sea prioritariamente descriptivo, el propósito último de esta exploración será analizar las condiciones de posibilidad de un turismo que sea relevante a nivel ético y, al mismo tiempo, que permite sortear los inconvenientes teóricos y prácticos relacionados con la visión tradicional del vínculo entre ética y turismo.

Para profundizar en ello, este estudio se basará en las investigaciones recientes de dos filósofos utilitaristas, Peter Singer y Joshua Greene, quienes se alejan de las versiones convencionales del utilitarismo y de la ética normativa tradicional en general, por el hecho de que incorporan en sus investigaciones una perspectiva evolutiva de la moral. En suma, es un punto de vista de la 
ética que contempla los posibles aportes de investigaciones actuales provenientes de disciplinas como la neurociencia, la psicología experimental, la primatología o la biología evolutiva en el análisis de la moral humana ${ }^{2}$. Se trata, tal como se argumentará a lo largo de este trabajo, de enfoques centrados en el análisis de cuáles son las condiciones necesarias para el surgimiento de comportamientos de tipo moral.

En el primer apartado, antes de avanzar sobre las teorías de los filósofos, se expondrán tres perspectivas ligadas al turismo ético. Para analizarlas se considerará, ante todo, lo que será definido como la distinción entre anclajes individuales e institucionales. La misma permitirá obtener un panorama amplio, desde el nivel más particular al más general en esta disciplina, para así evidenciar algunos puntos problemáticos.

En el segundo apartado se avanzará con la perspectiva de Peter Singer, quien en su libro Salvar una vida (2012) analiza la diferencia que el vínculo personal directo y el vínculo indirecto genera en los comportamientos morales individuales, así como también se expondrán los argumentos centrales de Joshua Greene sobre las diferencias entre el comportamiento moral intragrupal y el inter-grupal o, en sus términos, entre el vínculo Yo-Nosotros y el Nosotros-Ellos a nivel evolutivo (2013).

Finalmente, en el tercer apartado, luego de desarrollar las características principales de los enfoques individual e institucional del turismo ético, y de haber expuesto las investigaciones de Singer y Greene, se ahondará en el argumento principal de este trabajo, en el cual se propondrá una visión alternativa a la tradicional del vínculo entre ética y turismo.

\section{ANCLAJE INDIVIDUAL E INSTITUCIONAL: TRES PERSPECTIVAS DEL TURISMO ÉTICO}

Esta sección se focalizará en los aportes y problemas de las tres perspectivas más recurrentes en la literatura relacionada con el turismo ético: el PPT, el turismo sustentable y el turismo voluntario. En primer lugar, el PPT propone, en términos generales, incluir la reducción de la pobreza en la agenda del turismo. Según Ashley et al. (2001, 2), la actividad buscada puede definirse

2. Para una introducción a la relevancia ética en particular y filosófica en general de la teoría de la evolución biológica véase, por ejemplo, Dennett, 1999; Joyce, 2006; Changeux, 2008; Lariguet, 2014; Zavadivker, 2016; Smith, 2017; Pérez Zafrilla, 2017; Braicovich, 2019; Suárez-Ruíz, 2020. 
como un turismo que genera beneficios netos para los menos favorecidos en las comunidades locales. A su vez, el PPT centraría su foco sobre todo en los países en vías de desarrollo, en los cuales el crecimiento de la industria turística debería ser acompañado por una reducción de la pobreza (Holden, 2008, 136). Los códigos de ética internacionales vinculados al turismo, aunque suelen incluir aspectos del turismo sustentable, generalmente poseen esta perspectiva como prioritaria.

Según Mitchell y Ashley (2010), existen tres modalidades en las que el turismo puede impactar en la pobreza. En primer lugar, puede poseer una 'influencia directa'. En esta modalidad se incluiría la posibilidad de crear oportunidades de empleo a través de la industria turística, tanto para individuos particulares como para pequeñas y medianas empresas, y el beneficio de las mejoras en infraestructura requeridas para el desarrollo del turismo, como por ejemplo la mejora en las comunicaciones, hospitales, agua potable o servicios como la energía eléctrica. En segundo lugar, habría una 'influencia secundaria' con un énfasis en los efectos indirectos del desarrollo turístico. Según los autores, esta ocurre cuando una inversión vinculada al turismo posee influencia en la macroeconomía, como por ejemplo la creación de demanda en el ámbito de la construcción, la agricultura o la producción artesanal, y donde su impacto puede extenderse más allá de la región turística particular (diferente de la influencia directa). Por último, también puede ocurrir una 'influencia dinámica'. Esta tercera modalidad se relaciona con el influjo a largo plazo que puede generar la industria turística, tanto en la estructura económica como en la cultura local. Ejemplos de ello son la integración de las mujeres en las redes económicas, el acceso a la educación formal y el ascenso social generado por el desarrollo de infraestructura, o el crecimiento macroeconómico sostenido.

Las principales críticas que se le han realizado a la perspectiva PPT son dos. En primer lugar, el hecho de que las múltiples definiciones existentes del concepto 'pobreza' (p. ej., Griffith, 2003; United Nations, 2000; World Bank, 2005) dificultan hablar unívocamente de un sólo enfoque pro-poor basándolo exclusivamente en análisis estadísticos cuantitativos (Mowforth y Munt, 2016). En segundo lugar, y quizás la principal, que se trata de un punto de vista que descuida, e incluso puede llegar a reproducir, el problema económico de fondo, a saber, las desigualdades estructurales entre los países desarrollados y los países en vías de desarrollo (p. ej., Chok et al., 2007; Scheyvens, 2007; Schilcher, 2007). 
La segunda perspectiva ético-social de la teoría del turismo emparentada con el PPT es el turismo sustentable. Este enfoque surge de la corriente económico-social del desarrollo sustentable, definido como una concepción del desarrollo económico que contempla las necesidades del presente sin comprometer las de las generaciones futuras (WCED, 1987, 49). En la agenda del turismo sustentable coincide el énfasis en la reducción de la pobreza con la preocupación medioambiental, esto es, la búsqueda de que el desarrollo económico no vaya de la mano de la explotación desmedida de los recursos naturales (Hall, 2007, 1). A su vez, en su búsqueda de un turismo basado en un balance entre naturaleza y cultura (Weaver, 2001; Fennell, 2003), se opone al turismo de masas convencional (término en el cual se profundizará más adelante).

Una perspectiva derivada del turismo sustentable, con un fuerte anclaje en el comportamiento individual más que en las características de la estructura macroeconómica, es el turismo basado en el decrecimiento (degrowthinspired tourism) cuyo argumento principal es que "el cambio sostenible sólo puede lograrse siguiendo los principios principales del decrecimiento, que están asociados a los límites del crecimiento económico y a la economía de estado estacionario, y movimientos como la vida simple, la sencillez del voluntariado y el movimiento lento" (Andriotis, 2018, 17)

Como puede verse, el PPT y el turismo sustentable se oponen hasta cierto punto, por el hecho de que el PPT no posee como principio fundamental el poner límites al desarrollo económico. Es más, un desarrollo sostenido podría ser comprendido como un componente importante en la reducción de la pobreza de las comunidades locales. Este supuesto sería aún más inaceptable para los defensores del degrowth-inspired tourism. Tal como afirma Andriotis, el teórico citado en el párrafo anterior y defensor del decrecimiento, "mientras que en la era de la globalización se suponía que el turismo ofrecería grandes oportunidades a las comunidades pobres, siendo que las fuerzas de libre mercado habrían hecho su 'magia', se ha demostrado que en la mayoría de ellas los ricos se han enriquecido y los pobres se han vuelto relativamente más pobres" (2018, ix). Ahora bien, en general el PPT suele ir de la mano con la búsqueda de sustentabilidad, dado que a largo plazo el crecimiento económico desmedido termina siendo perjudicial también para los menos favorecidos (Yu y Duverger, 2019, 83).

La tercera perspectiva es el turismo voluntario o 'volunturismo' (voluntourism) (Vrasti, 2013, 1), el cual puede ser definido como "utilizar tiempo e ingresos discrecionales para viajar fuera de la esfera de actividad regular con 
el fin de ayudar a personas en necesidad" (McGehee y Santos, 2005, 760). Los sujetos comprometidos en esta práctica turística podrían sostener entre sus convicciones personales alguna de las dos perspectivas anteriores, dado que no entrarían en contradicción con su afán voluntarista, es decir, podrían funcionar como perspectivas macro de su actividad a nivel micro. Justamente, esta tercera perspectiva ético-social posee su acento puesto en la voluntad del individuo particular. Tal como afirma la investigadora Wanda Vrasti: "el éxito de la experiencia depende de la 'actitud personal'. Se alienta a los voluntarios a mostrar una mentalidad abierta y humildad hacia la cultura y las personas locales" $(2013,5)$. Al igual que el PPT, el turismo voluntario suele ir de la mano de perspectivas vinculadas al desarrollo sustentable, es decir, a enfoques críticos del desarrollo económico desmedido (Butcher y Smith, $2015,10)$. Este punto en común de las tres perspectivas del turismo ético será retomado más adelante para analizar su aplicabilidad en un contexto global donde el turismo de masas, el cual dista de coincidir con las máximas del desarrollo sustentable, es el fenómeno turístico que predomina.

En resumen, teniendo en cuenta sus particularidades, las tres perspectivas del turismo ético recién desarrolladas poseen en común un desafío, en mayor o menor grado, con mayor o menor éxito, a la política económica existente del turismo asentado exclusivamente en su dimensión industrial (Holden, 2013, 136). Una diferencia importante para el desarrollo que sigue es el nivel en el cual se concentra cada una: el enfoque PPT posee el énfasis puesto en el nivel institucional y macroeconómico; el turismo voluntario en la voluntad y el comportamiento individual; y el turismo sustentable, incluyendo aquí también el degrowth-inspired tourism como una ramificación contemporánea, bascula entre un anclaje individual y uno institucional.

Más allá de la relevancia que puedan tener estas corrientes actuales, hay un componente fundamental en la práctica turística, ya mencionado en la introducción, que dificulta la efectividad de las tres perspectivas ético-sociales, y es la dimensión complementaria y constitutiva del turismo: la industrial.

Un problema recurrente de los enfoques más generales, es decir, de los que hacen hincapié en el nivel institucional a través de, por ejemplo, códigos de ética que contemplen una perspectiva PPT o de desarrollo sustentable, es que no pocas veces pierden conexión con lo que sucede en lo concreto. Para graficar esto de manera directa, resulta pertinente la perspicaz imagen de Lovelock y Lovelock cuando evidencian la tensión existente entre el 'turista responsable' y el 'turista deseable' en la actividad turística concreta, afirmando que las instituciones turísticas generalmente se adecúan, en términos 
formales, a las características de "Mr Right, pero Mr Wrong es uno de los que más gasta (...). Entonces, el Sr. Wrong, si bien no es 'ético' o 'responsable', quizás sea el turista ideal de la industria del turismo (al menos desde una perspectiva económica a corto plazo)" (2013, 359). Nuevamente, el núcleo del problema parece ser la tensión entre la dimensión industrial y la éticosocial del turismo.

Otros son los problemas que surgen en los enfoques centrados en el individuo, como en el turismo voluntario o el degrowth-inspired tourism, y es la posibilidad de que los criterios éticos se alejen o, incluso, vayan en desmedro del alcance moral y político del turismo. En palabras de Butcher:

Contrariamente a las afirmaciones de que el turismo ético es una política progresista, en realidad lo que hace es disminuir la política en dos sentidos. Primero, la política queda de lado cuando las cualidades personales reemplazan las categorías políticas en el discurso del desarrollo público. Esto refleja y refuerza el vaciamiento de la esfera pública y, por lo tanto, reduce la agencia política y moral. Segundo, el turismo ético es una perspectiva particular que se disfraza, a través de términos como cuidado y responsabilidad, como una ética universal para todos. Como tal, limita la discusión sobre las diferentes opciones posibles del desarrollo. Se transforma, por tanto, en una etiqueta asfixiante para quien pretenda discutir elecciones políticas y sociales reales. De hecho, es un problema para cualquiera que quiera ampliar los horizontes políticos y restablecer un foro público para la política de desarrollo, más allá de las declaraciones moralistas sobre cómo los individuos eligen pasar sus vacaciones. $(2014,26)$

Según el teórico, cuando la cuestión ética se focaliza exclusivamente en los individuos, se descuida el nivel más amplio y, al mismo tiempo, más concreto de las problemáticas sociales. Aunque Butcher parece dirigir sus dardos hacia los desarrollos anclados en el individuo, su crítica puede extenderse también al anclaje institucional, dado que el énfasis en criterios abstractos que pierden los contextos particulares incurre en el mismo problema: el descuido de la dimensión político-social.

La advertencia de este pensador, así como las de Lovelock y Lovelock, permiten evidenciar que resulta imprescindible hallar visiones alternativas del vínculo entre ética y turismo que escapen, por un lado, a la dependencia de la voluntad de individuos particulares y, por otro lado, de prescripciones éticas limitadas al ámbito de lo formal. Para realizar establecer un punto de partida de esta búsqueda, en la próxima sección se desarrollarán los aspectos principales de la moral según Peter Singer quien, basándose en el marco 
teórico de Paul Slovic, distingue entre un sistema afectivo y un sistema deliberativo en los comportamientos morales. A su vez, se expondrán algunos conceptos relevantes desarrollados por el filósofo y neurocientífico Joshua Greene, vinculados a lo que él denomina como 'tribus morales'.

\section{La moral en clave evolutiva: Singer y Greene}

En su libro Salvar una vida (2012), Singer plantea un ya famoso experimento mental. En él, el filósofo presenta una situación hipotética en la que un hombre caminando por un parque se encuentra con un niño ahogándose en un estanque. Si bien posee un traje italiano de más de 500 dólares, esta persona no duda en rescatarlo aunque se arruine su lujosa ropa. Singer resalta que una acción diferente, en la que el hombre priorice su traje por sobre la vida del niño, nos resultaría moralmente monstruosa. A partir de allí, el filósofo se plantea la siguiente pregunta: ¿por qué es moralmente aceptable gastar $\$ 500$ en un traje si se podría usar ese dinero para salvar la vida de un niño haciendo una donación a una organización de ayuda internacional? No resulta coherente el hecho de que salvar a un niño ahogándose sea moralmente obligatorio, pero moralmente opcional si se trata de un niño desconocido.

Complementariamente, en dicho texto Singer menciona una serie de investigaciones que sustentan el siguiente hecho: los humanos generan un vínculo empático mucho más fuerte con una persona de carne y hueso que con la mención abstracta de la existencia de alguien necesitado en algún rincón del mundo. Uno de los conceptos utilizados por el filósofo para dar cuenta de este fenómeno es el 'efecto de la víctima identificada' (Singer, 2012, 65). Desde este se comprende que, en el momento en que un individuo es interpelado sobre el estado de vulnerabilidad de un otro, la empatía directa cumple un rol fundamental. Es decir, es mucho más probable que un individuo realice una donación a una organización caritativa si puede identificar su destino con una persona específica, con un rostro en particular, y no sólo con una estadística. En otros términos, es más probable que las personas donen cuando se les muestra un niño en particular, y menos probable cuando se les menciona una situación abstracta como la del hecho de que "la escasez de alimentos en Malawi afecta a más de 3 millones de niños” (Singer, 2012, 65).

Para ahondar en las características de esta particularidad de la moral humana, Singer, siguiendo al psicólogo Paul Slovic, argumenta que existen dos tipos de sistemas involucrados en la toma de decisiones: el 'sistema afectivo' 
y el 'sistema deliberativo'. El afectivo estaría relacionado con las reacciones emocionales, con la acción inmediata y poco premeditada, y el deliberativo con procesos conscientes, racionales, los cuales requieren de más tiempo para evaluar una estrategia de acción. Al poner en relación estos dos tipos de sistemas con el efecto de la víctima identificada, se comprende que ante la presencia de un individuo necesitado, dicho sujeto "arrastra nuestras emociones" (Singer, 2012, 66). Es decir, al presenciar de manera directa una situación en la que la vida de un individuo particular corre riesgo, se pone en funcionamiento el sistema afectivo, haciendo que la persona interpelada se vea empáticamente involucrada en la suerte de ese individuo. Por el contrario, las decisiones influidas por "hechos abstractos, transmitidos mediante palabras y cifras, no varía sustancialmente en función de las emociones" (Singer, 2012, 67). Esto es, al estar el sistema deliberativo vinculado a una reflexión más pausada y calculada, las emociones influyen menos, disminuyendo con ella la influencia de la empatía que la persona puede sentir para con los otros.

Entonces, el sistema deliberativo, en los casos en que se tiene noticia de penurias vividas por personas desconocidas en un lugar distante, generaría una suerte de sosegamiento del sistema afectivo. Como consecuencia, en los momentos en que predomina el sistema deliberativo (la 'razón') hay menos probabilidad de que las personas realicen donaciones, o estén dispuestas a ayudar a un otro que no conocen. Según esto, en términos generales, mientras más ajena resulte la situación de una persona, menos preocupación se sentirá por ella. En este tipo de casos, por más mención que se haga de estadísticas o conceptos abstractos, estos parecen no contribuir al vínculo empático, dado que dicha información se mantiene en un nivel racional, es decir, donde predomina el sistema deliberativo por sobre el afectivo.

A su vez, Singer afirma que existe una disminución gradual de la preocupación social a medida que el otro necesitado se aleja de la cotidianidad del individuo interpelado. Por tanto, podría hablarse de al menos tres niveles de vínculo empático: en primera instancia se encontraría la familia; luego los que comparten nuestra identidad cultural; y recién a partir de allí, personas que se encuentren más allá de ese límite. Esto se evidencia estadísticamente: la ayuda brindada a otros países es una mínima parte comparada con la que las personas ofrecen al propio país (Singer, 2012, 68). De modo que el sistema deliberativo no es el único obstáculo en la solidaridad para con los otros, ya que la prioridad puesta en el grupo más próximo, favorecida por el sistema afectivo, también jugaría un rol fundamental en la decisión de donar o no. 
Según el filósofo, esta particularidad denominada 'provincianismo', se relacionaría con el trayecto evolutivo de la especie humana:

Nuestra especie ha necesitado millones de años de evolución para convertirse en mamíferos sociales con una prole que requiere la atención de sus progenitores durante un largo período de tiempo. La mayor parte de esos millones de años, los progenitores que no atendían a sus criaturas durante sus años de dependencia tenían pocas probabilidades de trasmitir sus genes. De ahí que nuestra inquietud por el bienestar de los demás suela limitarse a nuestros parientes, a aquellos con quienes establecemos lazos de cooperación y, tal vez, a los miembros de nuestra pequeña comunidad tribal (Singer, 2012, 68).

La disminución gradual de la preocupación por los otros, entonces, estaría relacionada con la característica 'tribal' de las primeras comunidades humanas, en las cuales la preocupación por sus más cercanos les permitió conservar la vida a lo largo de las generaciones. Esa característica no habría quedado atrás en el trayecto evolutivo, sino que, tal como sostiene el filósofo y neurocientífico Joshua Greene, influye aún hoy en los grupos humanos.

En su libro Moral tribes (2013), Greene, al igual que Singer, se basa en una teoría de proceso dual similar a la de Slovic y analiza cómo este procesamiento dual influye en la moral humana ${ }^{3}$. Según el filósofo, es preciso tener en cuenta que, en el marco de la evolución humana, no habría existido un único momento fundacional del lazo cooperativo, sino que la moral se habría constituido como un fenómeno que fue emergiendo y asentándose gradualmente, generación tras generación (Greene, 2013, 20). A su vez, siendo que la cooperación se convirtió en un vínculo fundamental para garantizar la supervivencia, el cuidado de los otros que pertenecen a nuestro grupo -la 'moral intra-grupal'- habría surgido, al menos en términos generales, como un impulso emocional, pre-reflexivo, más que como uno detenidamente razonado (Greene, 2013, 20). Por tanto, se trataría de un proceso filogenéticamente antiguo en el cual predomina, siguiendo la distinción de Slovic, más el sistema afectivo que el deliberativo. De modo que, al menos en términos generales, el Nosotros es, antes que el Yo, la prioridad en el comportamiento

3. Sumados a Greene y Slovic, otros dos reconocidos investigadores que se basan en las teorías de proceso dual (dual-process theories) para analizar las características de las decisiones y acciones humanas son Daniel Kahneman (2012) y, de particular relevancia para la cuestión moral, Jonathan Haidt (2001). Para una introducción a los debates actuales relacionados con estas teorías véase Brand, 2016. 
humano, por tratarse de una especie cuya evolución estuvo ligada a un fuerte vínculo social.

No obstante, el autor advierte que este vínculo intra-grupal consiste en una adaptación biológica que posee una consecuencia complementaria, dado que en el marco del proceso evolutivo que favoreció grupos humanos con fuertes vínculos cooperativos hacia su interior, no sucedió lo mismo con la relación inter-grupal $(2013,23)$. Es decir, aunque la moral fue favorecida en la evolución humana para achicar la distancia entre el Yo y el Nosotros, no estaría preparada para la relación Nosotros-Ellos, de allí que Greene hable de 'tribus morales'. Dicha particularidad sería otra de las variables que subyacen a la dificultad de darle relevancia moral a una situación en la que los que necesitan ayuda son individuos indeterminados (Greene, 2013, 258).

A partir de las afirmaciones de Greene podría llegarse a una conclusión pesimista: la ética inter-grupal es imposible. No obstante, de manera similar a Singer, el filósofo argumenta que los seres humanos tienen la posibilidad de exceder los límites de su moral 'por defecto', a través de aspectos como la cultura y la capacidad racional. En sus palabras:

(...) la moral puede hacer cosas para las que no evolucionó (biológicamente). Como seres morales, podemos tener valores que se oponen a las fuerzas que dieron origen a la moralidad. Para tomar prestada la famosa metáfora de Wittgenstein, la moralidad puede trepar la escalera de la evolución y luego patearla. (...) La moral es más que aquello para lo cual evolucionó. (Greene, 2013, 25)

Los seres humanos, entonces, tendrían la posibilidad de establecer una 'meta-moral' que permita, al menos en cierto grado, sobrepasar los límites de la moral intra-grupal.

En la sección siguiente se pondrán en relación los desarrollos de Greene y Singer con contenidos provenientes de la teoría del turismo, con el fin de avanzar sobre el objetivo expresado en la introducción, a saber, analizar una perspectiva alternativa del vínculo entre ética y turismo desde la cual pueda evidenciarse el potencial moral indirecto de esta actividad.

\section{Sobre El POTENCIAL MORAL INDIRECTO DEL TURISMO CULTURAL DE MASAS}

Para comprender cómo los desarrollos de Singer y Greene permiten echar nueva luz sobre el vínculo entre ética y turismo, primero es preciso introducir dos conceptos importantes aún no definidos: 'turismo de masas' y 'turis- 
mo cultural'. El primero se asienta en una "suma de actividades industriales y comerciales que producen bienes y servicios total o principalmente para consumo turístico" (Weaver y Lawton, 2002, 471). Se trata de una práctica correlacionada con un fuerte apoyo a la economía de libre mercado, donde predomina una regulación débil y cuyo fin es, ante todo, favorecer la rentabilidad a corto plazo en beneficio de los accionistas (Weaver, 2006, 43). Por tanto, la dimensión que impera aquí es la industrial, de allí que los teóricos ligados al desarrollo sustentable sean sumamente críticos con esta visión dominante en el turismo. Por otro lado, a nivel de la escala demográfica involucrada, una práctica opuesta al turismo de masas sería, por ejemplo, el turismo de nicho, caracterizado por actividades turísticas personalizadas (Novelli, 2005).

Respecto del turismo cultural, y siguiendo la definición de McKercher y $\mathrm{Du}$ Cros, podría ser caracterizado como una práctica turística especial en la cual la diversidad cultural conforma la base de la atracción o la motivación del viaje $(2002,4)$. Aunque antiguamente era considerado un lujo de la elite (Pack, 2006, 5), actualmente el turismo cultural se ha convertido en una actividad relativamente más accesible en términos económicos. En palabras de Tikhonova, Sangkyun y Butler:

En términos del gran volumen de personas que viajan para experimentar otras culturas, el turismo cultural también ha crecido hasta convertirse en el nuevo turismo de masas (Du Cros y McKercher, 2015). Algunos turistas consideran sus aventuras culturales mucho más seriamente que otros (Stebbins, 1996), pero aprender sobre lugares y personas distantes es una razón muy común para viajar, a menudo combinado con relajación y entretenimiento (Jovicic, 2016). $(2018,111)$

Ahora bien, es preciso tener en cuenta que, aunque el turismo cultural puede considerarse hoy un turismo de masas, este último no se limita al primero. Tal como afirma Lew $(2017,9)$, los objetivos primarios en el turismo de masas, según las estadísticas económicas, son la visita de parques temáticos, cruceros y shopping centers, donde el interés de conocer otras culturas es mínimo o nulo.

Según lo desarrollado, es posible afirmar que, a la luz de las perspectivas del turismo ético descriptas en la primera sección del desarrollo, el turismo de masas se manifiesta como un fenómeno en sí mismo negativo, dado que es el tipo de actividad turística que más alejado está de la dimensión éticosocial. Por tanto, al menos hasta cierto punto, el turismo cultural también lo sería, por el hecho de que es una práctica que se ha vuelto masiva y donde 
parece haber predominancia de la dimensión industrial por sobre la social. No obstante, más allá de la pertinencia de este tipo de críticas, hay un fenómeno concreto sumamente importante a la hora de hacer efectivas nuestras pretensiones moralizantes: el turismo de masas es un hecho.

Habiendo llegado a este punto del desarrollo, cabe preguntarse, en un contexto global donde el turismo masivo parece ser un fenómeno insoslayable, ¿dónde sería posible hallar una intersección entre la dimensión ético-social y el turismo de masas? Esta pregunta pareciese ser una aporía, dado que ninguna de las tres perspectivas del turismo ético presentadas en la primera sección evidencia poder considerar aspectos positivos en el turismo masivo.

Desde un anclaje individual, el 'volunturismo' o el degrouth-inspired turism serían ante todo casos excepcionales y personales que no pueden ser extendidos a niveles masivos. Tal como se mencionó en la primera sección, la mayor dificultad de las perspectivas del turismo ético con foco en el individuo es su dependencia de la voluntad particular de cada sujeto.

Por otro lado, las éticas turísticas centradas en el aspecto institucional parecerían, a primera vista, poder hacer convivir el turismo de masas con un comportamiento acorde a sus fines éticos, dado que en la práctica estos se manifiestan, ante todo, como una lista de prevenciones que no limitan demasiado la acción del turista. La herramienta más específica del anclaje institucional, y aquí es donde el turismo ético coincide con la ética del turismo, es el código de conducta. Dicha herramienta tiene como fin mejorar el comportamiento y aumentar la confianza del turista en el lugar nuevo, evitar conflictos entre turistas y anfitriones, y actuar como una herramienta de gestión de visitantes para reducir sus impactos negativos -objetivos característicos de la ética del turismo-(Roberts y Rognvaldson, 2001), pero también favorecer una toma de consciencia del turista respecto de las características sociales de la comunidad local -objetivo característico del turismo ético- (Schilcher, 2007, 64).

Ahora bien, el código de conducta, siendo que se impone en un contexto en el cual los individuos suelen priorizar la búsqueda de placer y que, en última instancia, se trata de una herramienta de coacción del comportamiento del turista, usualmente no es respetado cuando se presenta la oportunidad (Cole, 2007, 445). De modo que el aspecto ético del enfoque institucional parece diluirse en las situaciones concretas, sobre todo cuando se tiene en cuenta la fragilidad de su impacto en el turismo de masas.

Entonces, sumando lo anterior a las críticas ya desarrolladas en la introducción y la primera sección, ni el enfoque individual ni el enfoque institucional 
del turismo ético parecen procurar una complementariedad efectiva entre la dimensión ético-social y el turismo de masas. Esto representa un grave problema, por el hecho de que si el turismo masivo, siendo la práctica más extendida a nivel global de la actividad turística, debe considerarse como un aspecto de la dimensión industrial poseedor de una articulación pobre o, incluso, nula con la dimensión ético-social, entonces pareciese no haber otra salida que aceptar como inexorable la reducción de gran parte del turismo exclusivamente a su dimensión industrial.

A pesar del panorama negativo, tal como se sostendrá de ahora en más, existe una intersección, un ámbito de complementariedad entre el turismo de masas y la ética que puede ser hallado en el turismo cultural de masas. Es en este punto de coincidencia donde existe la posibilidad de hallar una concepción alternativa del vínculo entre turismo y ética, según la cual no es la ética la que aplica sus preceptos normativos a la actividad turística, sino que es el turismo en sí mismo el que se revela como una actividad potencialmente relevante en términos morales.

El argumento principal, tal como se adelantó en la introducción, reside en que la relación directa entre personas concretas pertenecientes a culturas sumamente diversas que caracteriza al turismo cultural de masas, genera condiciones favorables para la emergencia de un vínculo empático entre individuos de diferentes grupos, lo cual, tal como se desarrolló en el segundo apartado, es condición necesaria de los comportamientos morales. Esta potencialidad moral no se limitaría a la situación particular del breve vínculo entre el visitante y el local, sino que podría generar una empatía a mediano o, incluso, a largo plazo para con una otredad cultural.

Volviendo al enfoque de Singer, resulta evidente que a través del sistema deliberativo por sí sólo no sería posible favorecer este tipo de vínculos empáticos. Ejemplo de ello es el poco efecto que poseen las estadísticas en la respuesta moral. Ahora bien, más allá de las limitaciones del sistema deliberativo, el filósofo hace hincapié en su fundamental relevancia para las decisiones y acciones humanas. De no ser por este sistema, no existiría la posibilidad de evidenciar sesgos como el 'provincianismo' para así tomar precauciones para con él. A su vez, por ejemplo, gracias al sistema deliberativo podemos evidenciar el problema tras el experimento mental del niño ahogándose y, en consecuencia, buscar soluciones más efectivas para este tipo de influencias en nuestras convicciones morales intra-grupales. Esto no significa que se deba hallar el modo de eliminar todo efecto proveniente del sistema afectivo, sino, más bien, encontrar maneras de encauzar también su potencialidad moral. 
Tal como lo ejemplifican sesgos del sistema deliberativo como la imperturbabilidad ante las estadísticas, una vez que una persona tiene contacto directo con alguien en necesidad, es más probable que se comporte moralmente. Es decir, el sistema afectivo muestra ser una condición imprescindible para que emerja una preocupación moral efectiva. De modo que, para que surja un vínculo empático y, eventualmente, el comportamiento moral, sería necesario generar un feedback entre el sistema afectivo y el deliberativo que el turismo parece tener la potencialidad de favorecer.

Siguiendo a Greene, aunque el vínculo moral Nosotros-Ellos sea dificultoso, el visitar lugares de culturas diferentes a la propia podría favorecer un tipo de relación no considerado en sus desarrollos, a saber, el vínculo YoEllos. Siendo que el estar en un lugar desconocido suele precisarse la creación de vínculos cooperativos que permitan facilitar la convivencia en el territorio ajeno, al incursionar en nuevas culturas surge una suerte de 'Nosotros cultural temporario' durante lo que dura la visita al lugar desconocido. Ese Nosotros temporario sería una condición necesaria para que pueda generarse una tribu moral que, a pesar de su fugacidad, podría tener repercusiones a largo plazo en la persona visitante. Es decir, en otros términos, podría contribuir a considerar la otredad de un modo diferente al del distanciamiento que caracteriza al vínculo Nosotros-Ellos. De modo que, el turismo cultural de masas, al dar lugar a un entrelazamiento de individuos con valores culturales diferentes a los propios, tiene la potencialidad de favorecer experiencias de un Nosotros cultural temporario, el cual, a su vez, podría hacer emerger vínculos empáticos entre personas sumamente diferentes y desconocidas.

Ahora bien, ¿cómo sería posible favorecer ese tipo de experiencias empáticas desde el turismo cultural de masas? No se trata de que las agencias turísticas, con el fin de que surjan vínculos empáticos entre desconocidos, impongan normas del tipo “¡hágase amigo de un local!”. Resulta inverosímil que una empresa así tenga éxito masivo entre los turistas. Tal como lo indica el título de este trabajo, el turismo cultural de masas tiene un potencial moral indirecto, es decir, el vínculo empático buscado no puede ser impuesto. No obstante, sí podrían favorecerse las condiciones propicias para estimular la emergencia de este tipo de experiencias.

En términos hipotéticos, una de las políticas institucionales posibles que podrían ponerse en marcha para favorecer este potencial moral indirecto sería códigos de conducta que procuren un vínculo entre los turistas y los locales no limitado, por ejemplo, a rápidas transacciones comerciales. Es decir, para que esa potencialidad se haga efectiva, resultan necesarias ciertas condiciones 
previas que favorezcan la emergencia del feedbak entre el sistema deliberativo y el afectivo y, consecuentemente, el Nosotros cultural temporario. Más allá de esta sugerencia de orden prescriptivo, tal como se adelantó en la introducción, el propósito de este trabajo es caracterizar las condiciones de posibilidad para pensar un vínculo entre ética y turismo diferente al tradicional, por lo que quedará pendiente el avance sobre el aspecto normativo.

Antes de finalizar, se mencionarán tres críticas posibles a esta propuesta. En primer lugar, podría decirse, en sintonía con la crítica moral y política de Butcher mencionada más arriba $(2014,26)$, que no sólo hay una resignación a la existencia indeterminada de la degradación provocada por el turismo de masas, sino que se busca ver este fenómeno como algo sumamente positivo. En defensa de los argumentos expuestos es preciso resaltar nuevamente que el turismo de masas es un hecho, por tanto, si acaso se busca eliminar sus efectos nocivos esto sólo podría acontecer buscando estrategias concretas para disminuirlos de manera gradual. Desde este punto de vista, el turismo cultural de masas es una herramienta interesante para reflexionar sobre ello.

En segundo lugar, podría señalarse que la pretensión de ver una potencialidad moral en el turismo cultural de masas es, básicamente, una ingenuidad, esto es, otro intento moralizador inconducente en la teoría del turismo. Sobre esto resulta pertinente resaltar que es por ello que este trabajo se centró exclusivamente (o casi) en el enfoque descriptivo, con el fin de sentar las bases de un punto de partida cimentado en estudios científicos recientes. Es decir, la intención de este artículo es presentar el problema y trazar una posible vía de investigación que no pierda de vista las características psicológicas de los individuos concretos ni la dimensión industrial del turismo.

En tercer lugar, otra crítica podría ser dirigida a un punto ya señalado y es la brevedad del vínculo entre el turista y el local. Dicha crítica es sostenida por el teórico del turismo David Fennell, quien posee una perspectiva evolutiva de la moral similar a las de Greene y Singer, pero con la cual llega a una conclusión pesimista en relación con el turismo. Según Fennell, a la luz de la ética evolutiva y las teorías de proceso dual de la mente, se explica por qué "la cooperación no suele tener lugar en el turismo, ya que esta se funda en relaciones estables a largo plazo basadas en la reciprocidad y el altruismo" $(2019,159)$. El corto plazo inherente a la relación del turista y el local sería insuficiente, según el autor, para dar lugar a un vínculo cooperativo. Esto implicaría también, según lo citado, que los comportamientos de tipo moral rara vez tendrían lugar. Aún más, el engaño sería lo más común en este tipo de situaciones, por el hecho de que los individuos involucrados son 
conscientes de que es muy probable que no vuelvan a verse nunca más (Fennell, 2019, 159).

En defensa de lo aquí desarrollado, cabe señalar que este tipo de prioridad del engaño sobre la cooperación es propia del intercambio comercial, el cual es sólo un aspecto del amplio espectro de experiencias involucradas en la actividad turística. A su vez, existen estudios recientes que sostienen que no es el placer el sentimiento prioritario buscado por los turistas culturales, sino las experiencias de tipo moral. Un ejemplo relevante es el de los investigadores Pinna, Correia y Chiappa, quienes en las conclusiones de un reciente trabajo afirman que "valores como el de ser de mente abierta, tener un sentido de justicia social, sabiduría, lealtad, honestidad, responsabilidad, honrar a los padres y los ancianos, la cortesía y la amistad son las principales influencias de la moral en el turismo" $(2018,91)$. A su vez, su tesis fuerte sostiene que esta tendencia permitiría hipotetizar que "los valores morales, entendidos como universales, pueden explicar la felicidad compartida entre diferentes culturas, más allá de otros factores situacionales" $(2018,91)$.

Este trabajo muestra ser más acorde a las conclusiones optimistas de Singer y Greene, quienes afirman que, por un lado, a través de un feedback del sistema deliberativo y el afectivo sería posible favorecer los comportamientos morales y, por otro lado, que los seres humanos no estamos completamente limitados por la tendencia a favorecer la moral intra-grupal, sino que tenemos la posibilidad de generar estrategias que favorezcan una meta-moral que la exceda.

\section{Conclusiones}

Lo desarrollado explicita que, paralelamente a la ética del turismo y del turismo ético, también es posible hablar de una potencialidad moral del turismo cultural de masas. Dicha potencialidad no surge de una aplicación de preceptos éticos normativos preestablecidos a la actividad turística, sino que es inherente a las características de dicha actividad y puede ser aprovechada para encontrar nuevos caminos en los estudios éticos de la teoría del turismo.

Para concluir este trabajo cabe resaltar nuevamente que el objetivo de este fue, ante todo, analizar las condiciones de posibilidad de una vía alternativa a la concepción tradicional del vínculo entre ética y turismo, para así hallar maneras más efectivas de concretar su articulación. No es posible determinar a priori cuáles serían las condiciones suficientes para favorecer la moralidad 
en y a través de la actividad turística, pero, a partir de lo desarrollado, sí ha sido posible considerar cuales serían algunas condiciones necesarias que generalmente son pasadas por alto.

\section{Agradecimientos}

El autor agradece al Consejo Nacional de Investigaciones Científicas y Técnicas (CONICET, Argentina) y a la Universidad Nacional de La Plata por el apoyo financiero.

\section{REFERENCIAS BIBLIOGRÁFICAS}

ANDRIOTIS, K. (2018). Degrowth in tourism. Conceptual, theoretical and philosophical issues. Boston: CABI.

Ashley, C., Roe, D. y Goodwin, H. (2001). Pro-poor Tourism Strategies: Making Tourism Work for the Poor: A Review of Experience. Londres: Overseas Development Institute.

Ateljevic, I., Morgan, N. y Pritchard, A. (eds.) (2011). The Critical Turn in Tourism Studies: Creating an Academy of Hope. Londres: Routledge.

BRAICOVICH, R. (2019). "Hacia una filogénesis del sentido de justicia. Una revisión de la evidencia proveniente de la primatología”. Estudios de Antropología Biológica.

BRAnd, C. (2016). Dual-Process Theories in Moral Psychology. Tübingen: Springer.

BRouder, P. (2020). "Reset redux: possible evolutionary pathways towards the transformation of tourism in a COVID-19 world". Tourism Geographies, 1-7.

BuTCHER, J. (2003). The Moralisation of Tourism. Sun, sand... and saving the world? Londres: Routledge.

Butcher, J. (2014). “Moralizing Tourism: Personal Qualities, Political Issues”. En Mostafanezhad, Mary y Hannam, Kevin (eds.). Moral Encounters in Tourism. Burlington: Ashgate.

Butcher, J. y Smith, P. (2015). Volunteer Tourism The lifestyle politics of international development. Nueva York: Routledge.

Cheong, S., y Miller, M. (2000). "Power and tourism: a Foucauldian observation", Annals of Tourism Research, 27: 371-90.

СHOK, S., MACBETH, J. y WARREN, C. (2007). "Tourism as a tool for poverty alleviation: A critical analysis of 'pro-poor tourism' and implications for sustainability". Current Issues in Tourism 10 (2\&3), 144-165.

Changeux, J. P. (2008). Du vrai, du beau, du bien. Une nouvelle approche neuronale. Paris: Odile Jacob. 
E. JOAQUÍN SUÁREZ RUÍZ

ÉTICA Y TURISMO EN EL MARCO DE LAS 'TRIBUS MORALES': EL POTENCIAL MORAL INDIRECO DE LA ACTIVIDAD TURÍSTICA...

Cole, S. (2007). "Implementing and evaluating a code of conduct for visitors", Tourism Management 28 (2): 443-51.

Davis, J.B. (2001). "Commentary: Tourism research and social theory. Expanding the focus”. Tourism Geographies 3 (2), 125-34.

DennetT, D. (1999). La Peligrosa Idea de Darwin. Barcelona: Galaxia Gutenberg.

Fayos-Sola, E. y CoOper, C. (eds.) (2019). The Future of Tourism Innovation and Sustainability. Cham: Springer.

Fennell, D. (2019). “The Future of Ethics in Tourism”. En Fayos-Solà, Eduardo y Cooper, Chris (eds.). The Future of Tourism Innovation and Sustainability. Cham: Springer.

Fennell, D. (2006). "Evolution in tourism: The theory of reciprocal altruism and tourist-host interactions". Current Issues in Tourism, 9 (2), 105-124.

FenNell, D. (2003). Ecotourism. Routledge, London.

Gössling, S., ScotT, D., \& Hall, C. M. (2020). "Pandemics, tourism and global change: a rapid assessment of COVID-19". Journal of Sustainable Tourism, 1-20.

Greene, J. (2013). Moral tribes. Nueva York: The Penguin Press.

Griffith, M. (2003). Pro Poor Growth. London: CAFOD.

HAIDT, J. (2001). "The emotional dog and its rational tail: a social intuitionist approach to moral judgment”. Psychological Review, 108, 814-834.

Hall, M. (ed.) (2007). Pro-poor Tourism: Who Benefits? Perspectives on Tourism and Poverty Reduction. Toronto: Channel View Publications.

Higgins-Desbiolles, F. (2020a). "Socialising tourism for social and ecological justice after COVID-19”. Tourism Geographies, 1-14.

Higgins-Desbiolles, F. (2020b). "The "war over tourism”: challenges to sustainable tourism in the tourism academy after COVID-19”. Journal of Sustainable Tourism, 1-19.

Holden, A. (2008). Environment and Tourism (Second Edition). Nueva York: Routledge.

Holden, A. (2013). Tourism, Poverty and Development. Nueva York: Routledge.

Joyce, R. (2006). The evolution of morality. Londres: MIT Press.

Kahneman, D. (2012). Pensar rápido, pensar despacio. Buenos Aires: Debate.

Kozak, M. y Kozak, N. (eds.) (2018). Tourist Behavior, An Experiential Perspective. Cham: Springer.

LARiguet, G. C. (2014). “Ética, giro experimentalista y naturalismo débil”. Estudios de Filosofía Práctica e Historia de las Ideas, 16, 2014.

LEW, A. A. (2017). "Tourism planning and place making: Place-making or placemaking?” Tourism Geographies, 19(3), 448-466.

Lovelock, B. y Lovelock, K. (2013). The ethics of tourism: Critical and applied perspectives. Londres: Routledge.

Mcgehee, N. y Santos, C. (2005). "Social change, discourse, and volunteer tourism”, Annals of Tourism Research, 32 (3), 760-76. 
Mckercher, B. y du Cros, H. (2002). Cultural Tourism: The Partnership Between Tourism and Cultural Heritage Management. Nueva York: Hawthorn Press.

Mitchell, J. y Ashley, C. (2010). Tourism and Poverty Reduction: Pathways to Prosperity. Londres: Earthscan.

Mostafanezhad, M. y Hannam, K. (eds.) (2014). Moral Encounters in Tourism. Burlington: Ashgate.

MoufakkiR, O. (2012). "Of Ethics, Leisure and Tourism: The 'Serious Fun of Doing Tourism”'. En Moufakkir, Omar y Burns, Peter (eds.). Controversies in Tourism. Cambridge: CABI.

Moufakkir, O. y Burns, P. (eds.) (2012). Controversies in Tourism. Cambridge: CABI.

Mowforth, M. y Munt, I. (2016). Tourism and Sustainability: Development, Globalisation and New Tourism in the Third World (Fourth edition). Nueva York: Routledge.

Niewiadomski, P. (2020). "COVID-19: from temporary de-globalisation to a rediscovery of tourism?”. Tourism Geographies, 1-6.

Novelli, M. (ed.) (2005). Niche Tourism: Contemporary Issues, Trends and Cases. Oxford: Butterworth-Heinemann.

PACK, S. (2006). Tourism and Dictatorship Europe's Peaceful Invasion of Franco's Spain. Nueva York: Palgrave.

PéreZ Zafrilla, P. J. (2017). "Racionalismo y emotivismo en perspectiva neuroética”. Pensamiento. Revista de Investigación e Información Filosófica, 73, 575-579.

Pinna, M., Correia, A. y del Chiappa, G. (2018). "Being Good to Be Happy? The Influence of Moral Values on Tourist Happiness”. En Kozak, M. y Kozak, N. (eds.). Tourist Behavior An Experiential Perspective. Cham: Springer.

RoberTs, l. y RognVAldSON, G. (2001). "The roles of interpretation in facilitating access to and in the countryside". En Roberts, L. y Hall, D. (eds.). Rural Tourism and Recreation: Principles to Practice. Wallingford: CABI, pp. 92-7.

SCheyvens, R. (2007). "Exploring the tourism-poverty nexus". Current Issues in Tourism 10.

SCHilcher, D. (2007). "Growth versus equity: The continuum of pro-poor tourism and neoliberal governance". Current Issues in Tourism 10.

Singer, P. (2012). Salvar una vida. Madrid: Katz.

Smith, D. L. (ed.) (2017). How biology Shapes philosophy. Cambridge: Cambridge University Press.

Smith, M. y Duffy, R. (2004). The Ethics of Tourism Development. Nueva York: Routledge.

Suárez-Ruíz, E. J. (2020). “Aporías de la ética evolutiva: una revisión de la ética normativa como 'ilusión efectiva”". Artefactos, 9(2), 103-119.

Tearfund (2000). A Tearfund Guide to Tourism: Don't Forget your Ethics. London: Tearfund. 
TeO, P. (2003). "Striking a balance for sustainable tourism: Implications of the discourse on globalization”. Journal of Sustainable Tourism 10 (6), 459-474.

Tikhonova, D. y Kim, S. (2018). "Conceptualising Challenging Experiences and Post-Travel Culture Involvement”. En Kozak, M. y Kozak, N. (eds.). Tourist Behavior An Experiential Perspective. Cham: Springer.

United Nations (2000). A Better World for All. New York: UN.

VRASTI, W. (2013). Volunteer Tourism in the Global South Giving back in neoliberal times. Nueva York: Routledge.

Weaver, D. (2001). Ecotourism. Milton: John Wiley \& Sons.

Weaver, D. (2006). Sustainable Tourism: Theory and Practice. Nueva York: Elsevier. Weaver, D. y Lawton, L. (2002). Tourism Management (Second edition). Milton: John Wiley \& Sons.

World Commission for Environment and Development (WCED) (1987). Our Common Future. The Brundtland Report. Oxford: Oxford University Press, 1987.

World Bank (2005). World Development Report 2006: Equity and Development. Washington: The World Bank.

Yu, L. y Duverger, P. (2019). “Tourism and Economics: Technologically Enabled Transactions”. En FAyos-SolÀ, E. y CoOper, C. (eds.). The Future of Tourism Innovation and Sustainability. Cham: Spinger.

Zavadivker, M. N. (2016). "Una deuda pendiente en las ciencias humanas: la inclusión de los aportes de la genética y la biología evolucionista”. Ludus Vitalis, 23(44), 163-178. 